Kosana Stanetić, Brankica Marković, Gordana Tešanović, Suzana Savić, Mladen Šukalo, Zora Kremenović

Public Primary Health Care Center,

Banja Luka, B\&H

\title{
Assessment of risk for falls in elderly
}

\section{Abstract}

Introduction: Elderly persons have higher risk for falls, compared to younger population. Although no single risk factor causes all falls, a great deal of risk factors to which an individual is exposed, i.e. osteoporosis, lack of physical activity, impaired vision, usage of drugs, living settings etc, can be treated.

Objective: To investigate the risk for falls in elderly patients treated in $\mathrm{Fa}$ mily medicine teaching center (ECPM), Primary Health Care Center Banja Luka.

Method: This prospective study was conducted in June 2012. The study included 150 patients aged 65 years and older. Patients were chosen randomly. In study were included patients who have visited their family doctors on every of Mondays in June 2012. The Tinetti Gait and Balance Instrument was used to asses the risk for falls. Patients were examined to asses gait and balance according to Tinetti questionnaire, and supplementary questionnaire was created to record data about age, sex, chronic diseases and drugs that patients take.

Results: The study included 91 (60.7\%) female and 59 (39.3\%) male patients. The average age of patients was 74.71 years. $77(51.3 \%)$ were aged 65 to 75 years and $73(48.7 \%)$ were more than 75 years old. Results of Tinetti Gait and Balance Instrument showed that the risk for falls was high in 55 (36.7\%), moderate in $31(20.7 \%)$ and low in $64(42.7 \%)$ patients.

Conclusion: Approximately, one third of investigated patients had high risk for falls, what indicates that family doctors should be more involved in fall prevention in elderly and in constant educating of older adults and their families.

\section{Introduction}

Getting old is not a disease, but a process in which vital functions become slower and diminished. The process of getting old starts in young and middle age, and the consequences of getting old are visible in older age when a person becomes more and more susceptible to diseases and injuries ${ }^{1}$. It is considered that the old age is the age $\geq 65$ years. Besides chronology, as a basis for defining the old age, the change in social role (e.g. change in work pattern, retirement, adult status of their children or menopause) and the change in capabilities (e.g. presence of chronic diseases or physical disability) are also used. The change in social role is the most important for defining the old age ${ }^{2}$.
The growth of older population has significantly faster rate than the growth of total population globally. The proportion of elderly persons has tripled in period from 1950-2009. and will triple again by 2050 . In developed regions throughout the world the proportion of elderly persons has already exceeded the proportion of children in the community, and it is expected to exceed that of children two times by 2050 . Median age is approximately 13 years higher in developed countries than in undeveloped countries, and by 2050 . is expected to be approximately 20 years higher ${ }^{3}$.

As the number of elderly in general population is increasing, the number of falls in elderly is also increasing. Almost one third of elderly falls at least once in their lifetime. The annual prevalence of falls in persons older than 65 years is $28 \%$, and $15 \%$ of persons who fall is in a group of so called 
healthy older persons. The morbidity due to falls doubles with every decade of age: 50/100 000 in persons by age of 65 years, $150 / 100000$ by age of 75 years and 525/100 000 in persons older than 85 years. Approximately in $15 \%$ of falls a consequence is a hip fracture. Elderly often have difficulty in walking as a result of older age, illness, pain and inactivity, what can result in falls. One of five older persons has difficulty in walking. Thirty percent of persons older than 75 has difficulties with climbing the stairs, $40 \%$ cannot walk more than $1 \mathrm{~km}$, and $7 \%$ cannot walk without assistance of another person. On average, $30 \%$ of elderly fall at home at least once every year ${ }^{l}$.

The causes of falls could be exogenous and endogenous. Common endogenous causes of falls are sudden decrease of blood pressure, especially after taking drugs that decrease blood pressure, heart blocks that can also be caused by drugs, dizziness due to disorders of central nervous system, various mental disorders and psychotropic drugs, impaired vision, musculoskeletal disorders that cause difficulty in walking etc.

Although no single risk factor causes all falls, a great deal of risk factors to which an individual is exposed can be treated or managed, e.g. impaired vision, osteoporosis, usage of drugs, lack of physical activity, living environment conditions etc. ${ }^{4}$.

\section{Objective}

The aim of this study was to investigate the risk for falls in elderly patients treated in Family Medicine Teaching Center, Primary Health Care Center Banjaluka and to investigate the influence of chronic diseases and usage of drugs on risk for falls

\section{Method}

The research is a prospective study which included 150 patients aged 65 or more years, treated in Family Medicine Teaching Center, Primary Health Care Center Banjaluka. Patients were chosen randomly. The study was conducted by five family doctors, who examined and interviewed patients aged 65 and older who came for medical examination on four Mondays in June 2012. All patients included in the study gave their written consent for participating in the study. The Tinetti Gait and Balance Tool ${ }^{5}$ was used in the study as a questionnaire to assess gait and balance, and data from medical charts of investigated patients about the presence of chronic diseases and the usage of drugs that can cause falls were recorded in a supplementary questionnaire.

For the statistical analysis of obtained data, patients were divided in two age groups: one with patients 65 to 74 years old, and the other with patients aged 75 or more years
Tinetti Gait and Balance Tool

\section{BALANCE SECTION}

\begin{tabular}{|c|c|c|c|}
\hline Activity & & Description & Scoring \\
\hline $\begin{array}{l}\text { Sitting Balance } \\
\text { (Patient is seated in } \\
\text { hard, armless chair) }\end{array}$ & $\begin{array}{l}\text { a) } \\
\text { b) }\end{array}$ & $\begin{array}{l}\text { Leans or slides in chair } \\
\text { Steady, safe }\end{array}$ & $\begin{array}{ll}\text { a) } & 0 \\
\text { b) } & 1\end{array}$ \\
\hline 2. Rises from chair & $\begin{array}{l}\text { a) } \\
\text { b) } \\
\text { c) }\end{array}$ & $\begin{array}{l}\text { Unable to without help } \\
\text { Able, uses arms to help } \\
\text { Able without use of } \\
\text { arms }\end{array}$ & $\begin{array}{ll}\text { a) } & 0 \\
\text { b) } & 1 \\
\text { c) } & 2\end{array}$ \\
\hline 3. Attempts to rise & $\begin{array}{l}\text { a) } \\
\text { b) } \\
\text { c) }\end{array}$ & $\begin{array}{l}\text { Unable to without help } \\
\text { Able, requires more } \\
\text { than one attempt } \\
\text { Able to rise, one } \\
\text { attempt }\end{array}$ & $\begin{array}{ll}\text { a) } & 0 \\
\text { b) } & 1 \\
\text { c) } & 2\end{array}$ \\
\hline $\begin{array}{l}\text { 4. Immediate standing } \\
\text { balance (first } 5 \\
\text { seconds) }\end{array}$ & $\begin{array}{l}\text { a) } \\
\text { b) } \\
\text { c) }\end{array}$ & $\begin{array}{l}\text { Unsteady (staggers, } \\
\text { moves feet, trunk sway) } \\
\text { Steady but uses walker } \\
\text { or cane or other support } \\
\text { Steady without walker } \\
\text { or cane or other support }\end{array}$ & $\begin{array}{ll}\text { a) } & 0 \\
\text { b) } & 1 \\
\text { c) } & 2\end{array}$ \\
\hline 5. Standing balance & $\begin{array}{l}\text { a) } \\
\text { b) }\end{array}$ & $\begin{array}{l}\text { Unsteady } \\
\text { Steady but wide stance } \\
\text { (medial heels more than } \\
40 \mathrm{~cm} \text { apart) and uses } \\
\text { cane, walker or other } \\
\text { support } \\
\text { Narrow stance without } \\
\text { support }\end{array}$ & $\begin{array}{ll}\text { a) } & 0 \\
\text { b) } & 1 \\
\text { c) } & 2\end{array}$ \\
\hline $\begin{array}{l}\text { Nudged (subject at } \\
\text { maximum position } \\
\text { with feet as close } \\
\text { together as possible, } \\
\text { examiner pushes } \\
\text { lightly on subject's } \\
\text { sternum with palm } \\
\text { of hand } 3 \text { times) }\end{array}$ & $\begin{array}{l}\text { a) } \\
\text { b) } \\
\text { c) }\end{array}$ & $\begin{array}{l}\text { Begins to fall } \\
\text { Staggers, grabs, catches } \\
\text { self } \\
\text { Steady }\end{array}$ & $\begin{array}{ll}\text { a) } & 0 \\
\text { b) } & 1 \\
\text { c) } & 2\end{array}$ \\
\hline $\begin{array}{l}\text { 7. Eyes closed, subject } \\
\text { in position as in } 6 .\end{array}$ & $\begin{array}{l}\text { a) } \\
\text { b) }\end{array}$ & $\begin{array}{l}\text { Unsteady } \\
\text { Steady }\end{array}$ & $\begin{array}{ll}\text { a) } & 0 \\
\text { b) } & 1\end{array}$ \\
\hline 8. Turning 360 degrees & $\begin{array}{l}\text { a) } \\
\text { b) }\end{array}$ & $\begin{array}{l}\text { Discontinuous steps, } \\
\text { unsteady } \\
\text { Steady }\end{array}$ & $\begin{array}{ll}\text { a) } & 0 \\
\text { b) } & 2\end{array}$ \\
\hline 9. Sitting down & $\begin{array}{l}\text { a) } \\
\text { b) } \\
\text { c) }\end{array}$ & $\begin{array}{l}\text { Unsafe (misjudged } \\
\text { distance, falls into chair) } \\
\text { Uses arms or not a } \\
\text { smooth motion } \\
\text { Safe, smooth motion }\end{array}$ & $\begin{array}{ll}\text { a) } & 0 \\
\text { b) } & 1 \\
\text { c) } & 2\end{array}$ \\
\hline \multicolumn{3}{|l|}{ Balance score } & $/ 16$ \\
\hline
\end{tabular}




\section{GAIT SECTION}

Patient walks across room, first at his/her ,usual pace”, then at „rapid, but safe pace”, using usual walking aid (cane, walker)

\begin{tabular}{|c|c|c|c|c|c|}
\hline & Activity & & Description & & \\
\hline 10. & $\begin{array}{l}\text { Initiation } \\
\text { of gait } \\
\text { (Immediately } \\
\text { after told to } \\
\text { "go") }\end{array}$ & $\begin{array}{l}\text { a) } \\
\text { b) }\end{array}$ & $\begin{array}{l}\text { Any hesitancy or multiple } \\
\text { attempts } \\
\text { No hesitancy }\end{array}$ & $\begin{array}{l}\text { a) } \\
\text { b) }\end{array}$ & \\
\hline 11. & $\begin{array}{l}\text { Step length } \\
\text { and height }\end{array}$ & $\begin{array}{l}\text { a) } \\
\text { b) } \\
\text { c) } \\
\text { d) } \\
\text { e) } \\
\text { f) } \\
\text { g) } \\
\text { h) }\end{array}$ & $\begin{array}{l}\text { Right swing foot does not pass } \\
\text { left stance foot with step } \\
\text {-passes left stance foot - } \\
\text { Right foot does not clear floor } \\
\text { completely with step } \\
\text {-completely clears floor- } \\
\text { Left swing foot does not pass } \\
\text { right stance foot with step } \\
\text {-passes right stance foot - } \\
\text { Left foot does not clear floor } \\
\text { completely with step } \\
\text {-completely clears floor- }\end{array}$ & $\begin{array}{l}\text { a) } \\
\text { b) } \\
\text { c) } \\
\text { d) } \\
\text { e) } \\
\text { f) } \\
\text { g) } \\
\text { h) }\end{array}$ & $\begin{array}{l}0 \\
1 \\
0 \\
1 \\
0 \\
1 \\
0 \\
1\end{array}$ \\
\hline 12. & $\begin{array}{l}\text { Step } \\
\text { symmetry }\end{array}$ & & $\begin{array}{l}\text { Right and left step length are } \\
\text { not equal (estimate) } \\
\text { Right and left step length } \\
\text { appear equal }\end{array}$ & $\begin{array}{l}\text { a) } \\
\text { b) }\end{array}$ & $\begin{array}{l}0 \\
1\end{array}$ \\
\hline 13. & $\begin{array}{l}\text { Step } \\
\text { continuity }\end{array}$ & $\begin{array}{l}\text { a) } \\
\text { b) }\end{array}$ & $\begin{array}{l}\text { Stopping between steps } \\
\text { Steps appear continuous }\end{array}$ & $\begin{array}{l}\text { a) } \\
\text { b) }\end{array}$ & $\begin{array}{l}0 \\
1\end{array}$ \\
\hline 14. & Path & & $\begin{array}{l}\text { Marked deviation } \\
\text { Mild/moderate deviation or } \\
\text { uses walking aid } \\
\text { Straight without walking aid }\end{array}$ & & $\begin{array}{l}0 \\
1 \\
2\end{array}$ \\
\hline 15. & Trunk & & $\begin{array}{l}\text { Marked sway or uses walking } \\
\text { aid } \\
\text { No sway but flexion of knees } \\
\text { or back or spreads arms while } \\
\text { walking } \\
\text { No sway, no flexion, no use of } \\
\text { arms and no walking aid }\end{array}$ & $\begin{array}{l}\text { a) } \\
\text { b) } \\
\text { c) }\end{array}$ & $\begin{array}{l}0 \\
1 \\
2\end{array}$ \\
\hline & Walk stance & & $\begin{array}{l}\text { Heels apart } \\
\text { Heels almost touching while } \\
\text { walking }\end{array}$ & $\begin{array}{l}\text { a) } \\
\text { b) }\end{array}$ & $\begin{array}{l}0 \\
1\end{array}$ \\
\hline \multicolumn{4}{|c|}{ Gait score } & & $/ 12$ \\
\hline \multicolumn{4}{|c|}{ Total Score: Balance score + Gait score } & & $/ 28$ \\
\hline
\end{tabular}

\section{Scoring}

Score is obtained by summarizing points for Gait and Balance sections or total Gait and Balance scores. Scoring is done on a three point scale with a range on each item of $0-2$, with 0 representing the most impairment. The maximum score for Gait section is 12 points, while the maximum for
Balance section is 16 points, with a total maximum score for the overall Tinetti questionnaire of 28 points.

\begin{tabular}{|c|c|}
\hline \multicolumn{2}{|c|}{ Score Interpretation } \\
\hline Total Tinetti score & Risk for falls \\
\hline$\leq 18$ & high \\
\hline $19-23$ & moderate \\
\hline$\geq 24$ & low \\
\hline
\end{tabular}

Analyzed present chronic diseases were: hypertension, coronary artery disease, heart arrhythmias, heart failure, anxiety disorders, depression, psychosis, cerebrovascular diseases, epilepsy, Parkinson's disease, musculoskeletal disorders (osteoporosis, osteoarthritis, and spine problems), diabetes mellitus, renal failure, cancer, prostatic hyperplasia and impaired vision.

Analyzed drugs used by patients were: antihypertensive drugs and diuretics, antiarrhythmics, nitrates, antidepressives, antipsychotic drugs, benzodiazepines, antiepileptic drugs, antiparkinsonian drugs, insulin, oral hypoglycemic agents, alpha-blockers and non-steroidal anti-inflammatory drugs (NSAIDs). For statistical analysis of obtained data, investigated patients were divided in four groups considering total number of used systemic drugs: group of patients who did not use any drugs, and groups of patients who used $1-3$ drugs; 4 - 9 drugs; and 10 or more drugs.

In data processing different statistical proceedings were used. Descriptive analysis in a form of frequencies and percentages was used for sample analysis. We determined the difference between subjects considering risk for falls, sociodemographic variables (sex, age, number and type of used drugs) and diseases by $\chi^{2}$ test. We analyzed the difference between risk from falls and average number of drugs that subjects use by analysis of variance (ANOVA). Statistical significance is on level of $p<0.05$.

\section{Results}

The study included 59 (39.3\%) male subjects and 91 (60.7\%) female subjects (Graph 1), 73 (48.7\%) patients were older than 75 years and $77(51.3 \%)$ patients aged from 65 to 75 years (Graph 2).

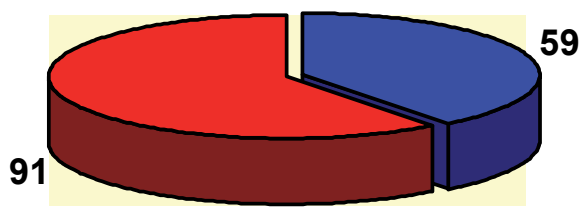

$\square$ male $\square$ female

Graph 1. Sex distribution of patients 


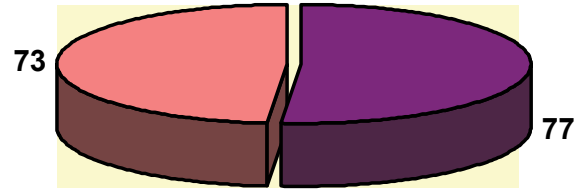

$\square 65$ to 75 years

77 Dover 75 years

Graph 2. Age distribution of patients

In investigated group of patients majority had one ore more chronic diseases. Most subjects had hypertension 132 $(88 \%)$, coronary artery disease $63(42 \%)$, heart arrhythmias $16(10.7 \%)$, stroke $12(8 \%)$, Parkinson's disease $10(6.7 \%)$, spine problems 42 (28\%), diabetes mellitus 37 (24.7\%), cancer $17(11.3 \%)$, impaired vision $26(17.3 \%)$, benign prostatic hyperplasia $20(13.3 \%)$ subjects. Other chronic diseases were found in a small number of patients (Table 1).

The most frequently used drugs in investigated group of patients were antihypertensive drugs in $123(82 \%)$, nonsteroidal anti-inflammatory drugs in $69(46 \%)$, diuretics in $66(43.3 \%)$ patients, nitrates in $43(28.7 \%)$, benzodiazepines in $50(33.3 \%)$, antiarrhythmics in $27(18 \%)$, oral hypoglycemic agents in $29(19.3 \%)$ subjects. Other drugs for therapy of chronic diseases were used by a small number of patients (Table 2).

Table 1. Presence of chronic diseases

\begin{tabular}{|l|c|c|}
\hline & $\begin{array}{c}\text { Number of } \\
\text { subjects }\end{array}$ & Percentage \\
\hline Cardiovascular diseases \\
\hline hypertension & 132 & $88.0 \%$ \\
\hline coronary artery disease & 63 & $42.0 \%$ \\
\hline heart failure & 6 & $4.0 \%$ \\
\hline heart arrhythmias & 16 & $10.7 \%$ \\
\hline Mental disorders and neurological diseases \\
\hline anxiety disorders & 3 & $2 \%$ \\
\hline depression & 5 & $3.3 \%$ \\
\hline stroke & 12 & $8.0 \%$ \\
\hline epilepsy & 3 & $2 \%$ \\
\hline Parkinson's disease & 10 & $6.7 \%$ \\
\hline Musculoskeletal disorders & \multicolumn{2}{|l|}{} \\
\hline hip osteoarthritis & 10 & $6.7 \%$ \\
\hline knee osteoarthritis & 18 & $12.0 \%$ \\
\hline spondylosis & 42 & $28.0 \%$ \\
\hline osteoporosis & 17 & $11.3 \%$ \\
\hline Other diseases & 3 & $2 \%$ \\
\hline renal failure & 17 & $11.3 \%$ \\
\hline cancer & 37 & $24.7 \%$ \\
\hline diabetes mellitus & 26 & $17.3 \%$ \\
\hline impaired vision & 20 & $13.3 \%$ \\
\hline benign prostatic hyperplasia & \\
\hline
\end{tabular}

Table 2. The most frequently used drugs

\begin{tabular}{|l|c|c|}
\hline \multicolumn{1}{|c|}{ Drug } & Number & Percentage \\
\hline antihypertensive drugs & 123 & $82.0 \%$ \\
\hline nitrates & 43 & $28.7 \%$ \\
\hline diuretics & 66 & $43.3 \%$ \\
\hline antiarrhythmics & 27 & $18.0 \%$ \\
\hline benzodiazepines & 50 & $33.3 \%$ \\
\hline antidepressives & 8 & $5.3 \%$ \\
\hline antiparkinsonian drugs & 9 & $6.0 \%$ \\
\hline antiepileptic drugs & 3 & $2.0 \%$ \\
\hline $\begin{array}{l}\text { non-steroidal anti- } \\
\text { inflammatory drugs } \\
\text { (NSAID) }\end{array}$ & 69 & $46.0 \%$ \\
\hline oral hypoglycemic agents & 29 & $19.3 \%$ \\
\hline insulin & 9 & $6.0 \%$ \\
\hline alpha blockers & 14 & $8.7 \%$ \\
\hline
\end{tabular}

In accordance with the number of chronic diseases in patients recruited in the study, a large number of patients used several types of drugs for treatment of chronic diseases. During duration of the study, the majority of subjects $85(56.7 \%)$ used four to nine drugs, one to three drugs used 54 (36.0\%) patients, drugs for chronic diseases did not use eight (5.3\%) patients, and three patients used at the same time ten or more drugs (Table 3).

Table 3. Number of used drugs

\begin{tabular}{|c|c|c|}
\hline Number of used drugs & Number of patients & Percentage \\
\hline 0 & 8 & $5.33 \%$ \\
\hline 1 to 3 & 54 & $36 \%$ \\
\hline 4 to 9 & 85 & $56.66 \%$ \\
\hline 10 and more & 3 & $2 \%$ \\
\hline
\end{tabular}

After analysis of the results from Tinetti questionnaire and summarizing points, the obtained data showed that the majority of investigated patients $64(42.7 \%)$ had low risk for falls, high risk had $55(36.7 \%)$ patients, and moderate risk had 31 patient (20.7\%) (Graph 3).

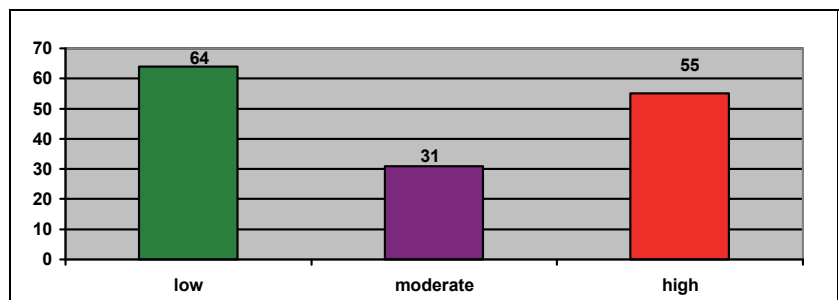

Graph 3. Risk for falls of all subjects (results of Tinetti questionnaire) 
Using additional statistical processing, risk from falls was analyzed in a relation to sex of subjects. Obtained results showed that female patients had statistically significantly $(p=0.037)$ higher risk for falls compared to male subjects. High risk for falls was present in $37(40.7 \%)$ female patients, and $18(30.5 \%)$ male subjects (Table 4$)$.

Table 4. Risk for falls considering sex of subjects

\begin{tabular}{|c|c|c|c|c|}
\hline \multirow{2}{*}{ Risk for falls } & \multirow{2}{*}{$\begin{array}{c}\text { Number } \\
\text { (N) and } \\
\text { percentage } \\
\text { of subjects }\end{array}$} & female & male & \multirow{2}{*}{$\boldsymbol{p}$} \\
\cline { 3 - 4 } & $\mathrm{N}$ & 32 & 33 & \\
\hline \multirow{2}{*}{ Low risk } & $\%$ & $35.2 \%$ & $55.9 \%$ & \multirow{2}{*}{0.037} \\
\cline { 2 - 4 } & $\%$ & 22 & 8 & \\
\hline \multirow{2}{*}{ Moderate risk } & $\mathrm{N}$ & $24.2 \%$ & $13.6 \%$ & \\
\cline { 2 - 4 } & $\%$ & 37 & 18 & \\
\hline \multirow{2}{*}{ High risk } & $\mathrm{N}$ & $40.7 \%$ & $30.5 \%$ & \\
\cline { 2 - 4 } & $\%$ & \multicolumn{2}{|c|}{ Sex } & \\
\hline
\end{tabular}

Results of our study revealed that older age significantly increases the risk for falls. Patients older than 75 years had statistically significantly ( $\mathrm{p}=0.000$ ) higher risk for falls compared to the group of subjects aged 65 to 75 years. High risk for falls had 39 (53.4\%) patients older than 75 years, and 17 (22.1\%) patients aged 65 to 75 years (Table 5).

Table 5. Risk for falls considering age

\begin{tabular}{|c|c|c|c|c|}
\hline \multirow{2}{*}{ Risk for falls } & \multirow{2}{*}{$\begin{array}{c}\text { Number } \\
\text { and } \\
\text { percentage } \\
\text { of subjects }\end{array}$} & \multicolumn{2}{|c|}{ Age } & \multirow{2}{*}{$\boldsymbol{p}$} \\
\cline { 3 - 4 } & $\begin{array}{c}\text { from } \\
\mathbf{6 5} \text { to 75 } \\
\text { years }\end{array}$ & $\begin{array}{c}\text { over 75 } \\
\text { years }\end{array}$ & \\
\hline \multirow{2}{*}{ Low risk } & $\mathrm{N}$ & 46 & 18 & \multirow{2}{*}{0.000} \\
\cline { 2 - 4 } & $\%$ & $59.7 \%$ & $24.7 \%$ & \multirow{2}{*}{} \\
\hline \multirow{2}{*}{ Moderate risk } & $\mathrm{N}$ & 14 & 16 & \\
\cline { 2 - 4 } & $\%$ & $18.2 \%$ & $21.9 \%$ & \\
\hline \multirow{2}{*}{ High risk } & $\mathrm{N}$ & 17 & 39 & \multirow{2}{*}{} \\
\cline { 2 - 4 } & $\%$ & $22.1 \%$ & $53.4 \%$ & \\
\hline
\end{tabular}

Considering number of used drugs, the greatest risk for falls was present in patients that use 10 or more drugs. Risk for falls statistically significantly $(\mathrm{p}=0.017)$ increases with the number of used drugs, so the group of patients that used 4 to 9 drugs had high risk for falls in $41.2 \%$ cases, in group of patients that used 1 to 3 drugs high risk for falls had $25.9 \%$ patients, and fewest patients who had high risk for falls was in group of patients who did not use drugs for therapy of chronic diseases (Table 6).
Table 6. Risk for falls considering number of used drugs

\begin{tabular}{|c|c|c|c|c|c|c|}
\hline \multirow{2}{*}{$\begin{array}{c}\text { Risk } \\
\text { for falls }\end{array}$} & $\begin{array}{c}\text { Number } \\
\text { and } \\
\text { percentage } \\
\text { of subjects }\end{array}$ & $\begin{array}{c}\text { Noes } \\
\text { not } \\
\text { take } \\
\text { drugs }\end{array}$ & $\begin{array}{c}\mathbf{1 - 3} \\
\text { drugs }\end{array}$ & $\begin{array}{c}\mathbf{4 - 9} \\
\text { drugs }\end{array}$ & $\begin{array}{c}\text { 10 or } \\
\text { more } \\
\text { drugs }\end{array}$ & \\
\hline \multirow{2}{*}{$\begin{array}{c}\text { Low } \\
\text { risk }\end{array}$} & $\mathrm{N}$ & 4 & 33 & 28 & 0 & \\
\cline { 2 - 6 } & $\%$ & $50.0 \%$ & $61.1 \%$ & $32.9 \%$ & $0.0 \%$ \\
\hline \multirow{2}{*}{$\begin{array}{c}\text { Moderate } \\
\text { risk }\end{array}$} & $\mathrm{N}$ & 0 & 7 & 22 & 1 & \multirow{2}{*}{0.017} \\
\cline { 2 - 6 } & $\%$ & $0.0 \%$ & $13.0 \%$ & $25.9 \%$ & $33.3 \%$ & \\
\cline { 1 - 5 } $\begin{array}{c}\text { High } \\
\text { risk }\end{array}$ & $\mathrm{N}$ & 4 & 14 & 35 & 2 & \\
\cline { 2 - 6 } & $\%$ & $50.0 \%$ & $25.9 \%$ & $41.2 \%$ & $66.7 \%$ & \\
\hline
\end{tabular}

Within our study we investigated the influence of the most common chronic diseases on the risk for falls. Results obtained by statistical processing revealed that hypertension does not increase risk for falls in investigated patients with statistical significance $(p=0.595)$. Risk for falls was not statistically significantly higher neither in patients with diabetes mellitus $(p=0.158)$ nor in patients with musculoskeletal disorders $(p=0.086)$. Chronic diseases that increased risk for falls in our patients with statistical significance were heart arrhythmias $(\mathrm{p}=0.004)$, heart failure $(\mathrm{p}=0.040)$ and coronary artery disease $(\mathrm{p}=0.027)$ (Table 7$)$.

In patients recruited in our study we investigated the influence of individual drugs on risk for falls. Obtained results revealed that risk for falls gets increased with statistical significance by diuretics $(\mathrm{p}=0.017)$ and non-steroidal anti-inflammatory drugs $(\mathrm{p}=0.025)$. Other the most frequently used drugs did not increase risk for falls in our patients with statistical significance: antihypertensive drugs $(\mathrm{p}=0.595)$, nitrates $(\mathrm{p}=0.162)$ and benzodiazepines $(\mathrm{p}=0.545)($ Table 8$)$. 
Table 7. Risk for falls considering presence of chronic diseases

\begin{tabular}{|c|c|c|c|c|}
\hline $\begin{array}{c}\text { Risk } \\
\text { for falls }\end{array}$ & $\begin{array}{c}\text { Number }(\mathrm{N}) \text { and } \\
\text { percentage of subjects }\end{array}$ & $\begin{array}{c}\text { Absence } \\
\text { of disease }\end{array}$ & $\begin{array}{c}\text { Presence } \\
\text { of } \\
\text { disease }\end{array}$ & $\mathrm{p}$ \\
\hline \multicolumn{5}{|c|}{ HYPERTENSION } \\
\hline \multirow{2}{*}{$\begin{array}{l}\text { Low } \\
\text { risk }\end{array}$} & $\mathrm{N}$ & 9 & 56 & \multirow{6}{*}{0.593} \\
\hline & $\%$ & $50.0 \%$ & $42.4 \%$ & \\
\hline \multirow{2}{*}{$\begin{array}{l}\text { Moderate } \\
\text { risk }\end{array}$} & $\mathrm{N}$ & 2 & 28 & \\
\hline & $\%$ & $11.1 \%$ & $21.2 \%$ & \\
\hline \multirow{2}{*}{$\begin{array}{l}\text { High } \\
\text { risk }\end{array}$} & $\mathrm{N}$ & 7 & 48 & \\
\hline & $\%$ & $38.9 \%$ & $36.4 \%$ & \\
\hline \multicolumn{5}{|c|}{ HEART ARRHYTHMIAS } \\
\hline \multirow{2}{*}{ Low risk } & $\mathrm{N}$ & 59 & 6 & \multirow{6}{*}{0.004} \\
\hline & $\%$ & $44.0 \%$ & $37.5 \%$ & \\
\hline \multirow{2}{*}{ Moderate risk } & $\mathrm{N}$ & 22 & 8 & \\
\hline & $\%$ & $16.4 \%$ & $50.0 \%$ & \\
\hline \multirow{2}{*}{ High risk } & $\mathrm{N}$ & 53 & 2 & \\
\hline & $\%$ & $39.6 \%$ & $12.5 \%$ & \\
\hline \multicolumn{5}{|c|}{ HEART FAШURE } \\
\hline \multirow{2}{*}{ Low risk } & \begin{tabular}{l|l}
$\mathrm{N}$ &
\end{tabular} & 65 & 0 & \multirow{6}{*}{0.040} \\
\hline & $\%$ & $45.1 \%$ & $0.0 \%$ & \\
\hline \multirow{2}{*}{ Moderate risk } & $\mathrm{N}$ & 29 & 1 & \\
\hline & $\%$ & $20.1 \%$ & $16.7 \%$ & \\
\hline \multirow{2}{*}{ High risk } & $\mathrm{N}$ & 50 & 5 & \\
\hline & $\%$ & $34.7 \%$ & $83.3 \%$ & \\
\hline \multicolumn{5}{|c|}{ CORONARY ARTERY DISEASE } \\
\hline \multirow{2}{*}{ Low risk } & $\mathrm{N}$ & 40 & 25 & \multirow{6}{*}{0.027} \\
\hline & $\%$ & $46.0 \%$ & $39.7 \%$ & \\
\hline \multirow{2}{*}{ Moderate risk } & $\mathrm{N}$ & 11 & 19 & \\
\hline & $\%$ & $12.6 \%$ & $30.2 \%$ & \\
\hline \multirow{2}{*}{ High risk } & $\mathrm{N}$ & 36 & 19 & \\
\hline & $\%$ & $41.4 \%$ & $30.2 \%$ & \\
\hline \multicolumn{5}{|c|}{ DIABETES MELLITUS } \\
\hline \multirow[t]{2}{*}{ Low risk } & $\mathrm{N}$ & 53 & 12 & \multirow{6}{*}{0.158} \\
\hline & $\%$ & $46.9 \%$ & $32.4 \%$ & \\
\hline \multirow[t]{2}{*}{ Moderate risk } & $\mathrm{N}$ & 19 & 11 & \\
\hline & $\%$ & $16.8 \%$ & $29.7 \%$ & \\
\hline \multirow[t]{2}{*}{ High risk } & $\mathrm{N}$ & 41 & 14 & \\
\hline & $\%$ & $36.3 \%$ & $37.8 \%$ & \\
\hline & IUSCULOSKELETAL DI & ORDERS & & \\
\hline Tom & $\mathrm{N}$ & 52 & 13 & \\
\hline Low risk & $\%$ & $48.1 \%$ & $31.0 \%$ & \\
\hline Mederate rick & $\mathrm{N}$ & 22 & 8 & 0006 \\
\hline Moderate nsk & $\%$ & $20.4 \%$ & $19.0 \%$ & 0.086 \\
\hline High risk & $\mathrm{N}$ & 34 & 21 & \\
\hline HIgn IISK & $\%$ & $31.5 \%$ & $50.0 \%$ & \\
\hline
\end{tabular}

Table 8. Risk for falls considering used drugs

\begin{tabular}{|c|c|c|c|c|}
\hline Risk for falls & $\begin{array}{l}\text { Number } \\
(\mathrm{N}) \text { and } \\
\text { percentage } \\
\text { of subjects }\end{array}$ & $\begin{array}{c}\text { Does not } \\
\text { use drugs }\end{array}$ & $\begin{array}{c}\text { Uses } \\
\text { drugs }\end{array}$ & $\mathbf{p}$ \\
\hline \multicolumn{4}{|c|}{ ANTIHYPERTENSIVE DRUGS } & \\
\hline \multirow{2}{*}{ Low risk } & $\mathrm{N}$ & 11 & 54 & \multirow{6}{*}{0.595} \\
\hline & $\%$ & $40.7 \%$ & $43.9 \%$ & \\
\hline \multirow{2}{*}{ Moderate risk } & $\mathrm{N}$ & 4 & 26 & \\
\hline & $\%$ & $14.8 \%$ & $21.1 \%$ & \\
\hline \multirow{2}{*}{ High risk } & $\mathrm{N}$ & 12 & 43 & \\
\hline & $\%$ & $44.4 \%$ & $35.0 \%$ & \\
\hline & DIURETIC & & & \\
\hline \multirow{2}{*}{ Low risk } & $\mathrm{N}$ & 44 & 21 & \multirow{6}{*}{0.017} \\
\hline & $\%$ & $51.8 \%$ & $32.3 \%$ & \\
\hline \multirow{2}{*}{ Moderate risk } & $\mathrm{N}$ & 11 & 19 & \\
\hline & $\%$ & $12.9 \%$ & $29.2 \%$ & \\
\hline \multirow{2}{*}{ High risk } & $\mathrm{N}$ & 30 & 25 & \\
\hline & $\%$ & $35.3 \%$ & $38.5 \%$ & \\
\hline & NITRATES & & & \\
\hline \multirow{2}{*}{ Low risk } & $\mathrm{N}$ & 51 & 14 & \multirow{6}{*}{0.162} \\
\hline & $\%$ & $47.7 \%$ & $32.6 \%$ & \\
\hline \multirow{2}{*}{ Moderate risk } & $\mathrm{N}$ & 18 & 12 & \\
\hline & $\%$ & $16.8 \%$ & $27.9 \%$ & \\
\hline \multirow{2}{*}{ High risk } & $\mathrm{N}$ & 38 & 17 & \\
\hline & $\%$ & $35.5 \%$ & $39.5 \%$ & \\
\hline & ENZODIAZEF & NES & & \\
\hline \multirow{2}{*}{ Low risk } & $\mathrm{N}$ & 44 & 21 & \multirow{6}{*}{0.545} \\
\hline & $\%$ & $44.0 \%$ & $42.0 \%$ & \\
\hline \multirow{2}{*}{ Moderate risk } & $\mathrm{N}$ & 22 & 8 & \\
\hline & $\%$ & $22.0 \%$ & $16.0 \%$ & \\
\hline \multirow{2}{*}{ High risk } & $\mathrm{N}$ & 34 & 21 & \\
\hline & $\%$ & $34.0 \%$ & $42.0 \%$ & \\
\hline \multicolumn{5}{|c|}{ / NON-STEROIDAL ANTI-INFLAMMATORY DRUGS } \\
\hline \multirow{2}{*}{ Low risk } & $\mathrm{N}$ & 42 & 23 & \multirow{6}{*}{0.025} \\
\hline & $\%$ & $51.9 \%$ & $33.3 \%$ & \\
\hline \multirow{2}{*}{ Moderate risk } & $\mathrm{N}$ & 17 & 13 & \\
\hline & $\%$ & $21.0 \%$ & $18.8 \%$ & \\
\hline \multirow{2}{*}{ High risk } & $\mathrm{N}$ & 22 & 33 & \\
\hline & $\%$ & $27.2 \%$ & $47.8 \%$ & \\
\hline
\end{tabular}

The majority of interviewed patients had one or more chronic diseases. The biggest number of subjects had one 21 (14.0\%), two $36(24.0 \%)$, three $45(30.0 \%)$, four $24(16.0 \%)$ or five 14 (9.3) chronic diseases (Table 9). 
Table 9. Number of chronic diseases per patient

\begin{tabular}{|c|c|c|}
\hline $\begin{array}{c}\text { Number of chronic } \\
\text { diseases }\end{array}$ & Number of patients (N) & Percentage (\%) \\
\hline 0 & 3 & 2.0 \\
\hline 1 & 21 & 14.0 \\
\hline 2 & 36 & 24.0 \\
\hline 3 & 45 & 30.0 \\
\hline 4 & 24 & 16.0 \\
\hline 5 & 14 & 9.3 \\
\hline 6 & 4 & 2.7 \\
\hline 7 & 2 & 1.3 \\
\hline 8 & 1 & 0.7 \\
\hline
\end{tabular}

The biggest number of interviewed patients (132 patients) had hypertension, and $60(45.5 \%)$ of these patients as a comorbidity had coronary artery disease, $16(12.1 \%)$ had heart arrhythmias, $12(9.1 \%)$ had cerebrovascular diseases (stroke), impaired vision had 18 (13.6\%), and diabetes mellitus had 37 (28.0\%) patients.

All patients with diabetes mellitus had hypertension as a comorbidity, 14 (37.8\%) had coronary artery disease, and 3 patients had heart arrhythmia.

Out of 10 patients with hip osteoarthritis, 9 had hypertension as a comorbidity, and 6 patients had spine problems. Patients with knee osteoarthritis had also hypertension in 17 (94.4\%) cases, coronary artery disease in $9(50.0 \%)$ cases, and spondylosis in $50 \%$ cases. Patients with spine problems had hypertension in $36(85.7 \%)$ cases, and coronary artery disease in $18(42.8 \%)$ cases.

Using further statistical processing (Pearson's coefficient of linear correlation) it is proved that patients that have more chronic diseases at the same time have statistically significantly higher risk for falls (Table 10).

Table 10. Correlation between risk for falls and the number of chronic diseases

\begin{tabular}{|c|c|c|}
\hline & Risk for falls & $\begin{array}{c}\text { Number of } \\
\text { chronic diseases }\end{array}$ \\
\hline Risk for falls & 1 & $0.314(* *)$ \\
\hline Number of chronic diseases & $0.314(* *)$ & 1 \\
\hline ** Correlation is significant on level 0.01 &
\end{tabular}

\section{Discussion}

Results of our study revealed that one third of subjects had high risk for falls. Statistically significantly higher risk for falls had female patients older than 75 years and patients who use several types of drugs in their regular medication regimen. The greatest risk for falls had patients suffering from cardiovascular diseases (heart arrhythmias, coronary artery disease and heart failure) and patients that use diuretics and NSAIDs.

The usage of drugs is constantly being associated with risk for falls. The greatest risk is associated with usage of psychotropic drugs and polypharmacy. It is proved that discontinuing of psychotropic drugs reduces fall rate. Although some doctors consider that the usage of selective serotonin reuptake inhibitors (SSRIs) is safer than the usage of tricyclic antidepressives, there is evidence that both groups of drugs equally increase the risk for falls ${ }^{4}$. Meta analysis of articles showed small, but consistent correlation of usage of psychotropic drugs in persons older than 60 years and risk for falls. Also, it pointed on the need of conducting large randomized controlled trials about the usage of drugs in older persons where the incidence of falls, as side effects of usage of drugs, would be followed prospectively ${ }^{5,6}$. A small number of patients recruited in our study suffer from depression and used antidepressives, so we did not compare the results of our study to the results of other studies. Benzodiazepines were used by $50(33.3 \%)$ patients, however no statistically significant higher risk for falls was found in these patients compared to patients that do not use benzodiazepines.

Similar meta analysis of articles followed the usage of cardiac drugs and analgesic drugs in persons older than 60 years and incidence of falls. It appeared that the usage of digoxin, antiarrhythmics type Ia and diuretics is weakly associated with falls in older adults. Other cardiac drugs and analgesic drugs did not show any association with risk for falls. Older adults who used more than three or four drugs were at increased risk of recurrent falls ${ }^{7}$. In our study more than 50\% of subjects take more than three drugs continuously. Risk for falls is increased in patients taking more drugs. These data give some room for engagement of family doctor in a sense of decreasing the number of drugs in regular medication regimen of our patients as a strategy for fall prevention.

Postural hypotension is associated with increased risk for falls. The most common causes are dehydration, usage of drugs or autonomic neuropathy. There are evidence from randomized controlled trials that treatment of postural hypotension, modification of usage of drugs that cause hypotension and behavioral interventions can decrease the risk for falls. Adequate management of these patients decreases the risk for falls ${ }^{4}$. Our study showed that hypertension was treated in majority of subjects, a lot of subjects had coronary artery disease and a small number of subjects had heart arrhythmias and heart failure. Patients with hypertension in our study mainly have some other chronic diseases, what increases the risk for falls.

In patients with stroke, Parkinson's disease or osteoarthritis, balance and gait are better reestablished if such patients do not have additional chronic diseases. Chronic dis- 
eases that appear to cause more severe disabilities and make recovering of gait and balance more difficult in such patients are chronic obstructive lung disease, heart failure, peripheral artery disease, diabetes, cancer that is not life thretening ${ }^{8,9}$. Musculoskeletal disorders in our subjects were not associated with increased risk for falls, but the usage of NSAIDs was. This can be explained with the fact that not all patients with musculoskeletal disorders use NSAIDs as a regular medication regimen. Considering a great number of side effects of these drugs, they are rarely recommended to be used for longer period of time.

In patients with diabetes, risk for falls is high because of the nature of their underlying disease. Tinetti test, together with tests of muscle strength, tests for neuropathy and foot deformity, could point to the risk for falls in diabetic patients, so that preventive measures could be undertaken on time ${ }^{10}$. Patients suffering from diabetes type 2, whether they live in rural or urban settings, share similar risk factors for chronic falling, namely similar comorbidities and take similar kind and number of drugs. Impaired balance in such patients can be assessed by Tinetti questionnaire; and it is the most frequently caused by impaired lower extremity protective sensation ${ }^{11}$. In our study, all patients suffering from diabetes had, as a comorbidity, hypertension and $37.8 \%$ also had coronary artery disease. Considering that these patients used drugs for treatment of their underlying disease and additionally drugs for treatment of comorbidities, they had a lot of drugs in their regular medication regimen what increased their risk for falls.
Many authors presented recommendations for fall prevention in older adults, as the most effective and the most efficient intervention. As preventive measures, health promotion, reviewing of drugs that patients take, physical training and insuring safety at home and in environment are mentioned $^{12,13}$. Other authors recommend professional training for recovering of gait and balance, gradual decrease of psychotropic substances intake (benzodiazepines, hypnotic drugs, antipsychotic drugs, antidepressives) and modifying the risky behavior ${ }^{14}$. As specific recommendations in the literature, advising patients to wear safe and comfortable footwear, slippers that do not slip, usage of light during the night and additional stairs, are mentioned. Drugs that all elderly patients take, including drugs they buy without prescription should be reviewed in a purpose of decreasing the number of drugs they use or modifying the dose. It is recommended that total number of used drugs is four or less, what considerably decreases the risk for falls ${ }^{15}$.

\section{Conclusion}

One third of investigated patients had high risk for falls. Chronic diseases that significantly increased the risk for falls in our patients were heart arrhythmias, heart failure and coronary artery disease. Drugs associated with high risk for falls were diuretics and NSAIDs. The role of family doctor in fall prevention is in constant educating of elderly and their families about living environment conditions, their disease or condition, prevention of complications and rational usage of drugs. 
Косана Станетић, Бранкича Марковић, Гордана Тешановић, Сузана Савић, Младен Шукало, Зора Кременовић

ЈЗУ Дом здравља Бања Лука,

Босна и Херцеговина

\section{Процена ризика од падова код старијих особа}

\section{Сажетак}

\section{ризик од падова,}

Кључне речи: старији пацијенти
Увод. Старије особе имају већи ризик од падова у односу на млађу популацију. Иако не постоји само један фактор ризика за падове код старијих, велики број фактора ризика којима је појединац изложен се може третирати: остеопороза, недостатак физичке активности, проблеми са видом, употреба лијекова, место становања и друго.

Циљ рада. Испитати ризик од падова код старијих пацијената лијечених у Едукативном центру породичне медицине $(E C P M)$ у Дому здравља Бања Лука.

Метод. Истраживање је проспективно, а проведено је током јуна 2012. године. У студију је укључено 150 пацијената старијих од 65 година. Пацијенти су изабрани методом случајног избора, на начин да су у студију укључени пацијенти који су се јавили на лекарски преглед сваког понедељка у јуну 2012. године код свог породичног лекара. Пацијентима је урађен преглед којим се испитивао ход и равнотежа према Tinetti упитнику, а у додатни анкетни упитник креиран за потребе овог истраживања уписивани су подаци о добу, полу, хроничним болестима и лековима које пацијенти користе.

Резултати. Истраживањем је обухваћен 91 (60.7\%) пацијенат женског и 59 (39.3\%) мушког пола, Просечне старости 74.71 година, 77 (51.3\%) је било у добу од 65 до 75 година, а 73 (48.7\%) старије од 75 година. Резултати Tinetti упитника за ход и равнотежу су показали да је висок ризик од падова имало 55 (36.7\%), умерен ризик 31 (20.7\%), а низак ризик 64 (42.7\%) пацијента.

Закључак. Једна трећина испитиваних пацијената је имала висок ризик од падова, што указује на значај породичног лекара у превенцији падова и сталном едуковању старијих особа и њихових породица. 


\section{Literatura}

\section{References}

1. Milanović P, Milošević D, Davidović M Padovi u starosti. Medicinska gerontologija. Beograd. Medicinski fakultet u Beogradu; 2007. [Falls in older age. Medical gerontology. Belgrade. School of Medicin in Belgrade]

2. World Health Organization. Definition of an older or elderly person. 2012.

3. United Nations. World Population Ageing 2009. New York, 2009.

4. Geriatrics Society Clinical Practice Guideline for Prevention of Falls in Older Persons, Summary of the Updated American Geriatrics Society/British, 2010.

5. Leipzig RM, Cumming RG, Tinetti ME Drugs and falls in older people: a systematic review and meta-analysis: I. Psychotropic drugs. J Am Geriatr Soc. 1999;47:30-9.

6. Di Fazio I, Franzoni S, Frisoni GB, Gatti S, Cornali C, Stofler PM, Trabucchi M. Predictive role of single diseases and their combination on recovery of balance and gait in disabled elderly patients. J Am Med Dir Assoc. 2006;7:208-11.
7. Leipzig RM, Cumming RG, Tinetti ME. Drugs and falls in older people: a systematic review and meta-analysis: II.Cardiac and analgesic drugs. J Am Geriatr Soc. 1999;47:40-50.

8. Gai J, Gomes L, Nóbrega Ode T, Rodrigues MP. Factors related to falls of elderly women residents in a community. Rev Assoc Med Bras. 2010;56:327-32.

9. Mazoteras Muñoz V, Abellan van Kan G, Cantet C, Cortes F, Ousset PJ, Rolland Y, Vellas B. Gait and balance impairments in Alzheimer disease patients. Alzheimer Dis Assoc Disord. 2010;24:79-84.

10. Macgilchrist C, Paul L, Ellis BM, Howe TE, Kennon B, Godwin J. Lower-limb risk factors for falls in people with diabetes mellitus. Diabet Med. 2010;27:162-8.

11. Conner-Kerr T, Templeton MS. Chronic fall risk among aged individuals with type 2 diabetes. Ostomy Wound Manage. 2002;48:28-35
12. Tinetti ME, Kumar C. The patient who falls: "It's always a trade-off". JAMA. 2010;303:258-66.

13. Das CP, Joseph S. Falls in elderly. Journal of the Indian Medical Association: 2005;103(3):136-140.

14. Evans JG. Drugs and falls in later life. Lancet 2003;361:448

15. Mary E, Tinetti MD. Preventing falls in elderly Persons. N Engl Med 2003;348:429.

Примљен • Received: 21/05/2013 Прихваћен • Accepted: 10/07/2013 